\title{
Consensus Disturbance Rejection with Disturbance Observers
}

\author{
Zhengtao Ding, Senior Member, IEEE
}

\begin{abstract}
This paper deals with consensus disturbance rejection of network-connected dynamic systems using disturbance observers. The control objective of consensus disturbance rejection is to achieve a common state trajectory for the networkconnected subsystems that are under deterministic disturbances. The difference from the existing disturbance rejection methods is that only the relative state information is used for disturbance rejection, and as a consequence of using the relative state information, only the part of the disturbances that affect the common trajectories will be rejected. The conditions for designing disturbance observers for consensus control are identified for networked-connect multi-agent systems. Certain features of the individual subsystems are analysed for possible implementation of disturbance-observer based rejection, and the disturbance observers are designed based on the relative state information obtained from the neighbouring subsystems under different network connections. When the network connectivity is available for the disturbance observer design, consensus disturbance rejection is achieved for a directed network with a spanning tree. A fully distributed consensus disturbance rejection design is presented for an undirected network with the use of adaptive parameters for the estimation of the unknown network connectivity. Disturbance observers are also proposed for disturbance rejection in the leader-follower consensus control.
\end{abstract}

Index Terms-Consensus control, Disturbance Rejection, Disturbance Observer, Adaptive Control

\section{INTRODUCTION}

Disturbance rejection has been one of the fundamental control design problems which has tremendous applications in aerospace, robotics, automation, process engineering, and various industries. Due to its important applications in diverse areas, disturbance rejection may appear under various names, such as anti-disturbance, disturbance suppression, disturbance attenuation, and output regulation etc, in addition to disturbance rejection [1], [2], [3], [4], [5], [6]. For the rejection of deterministic disturbances such as constants and sinusoidal disturbances, the controller must have the ability to generate the desired input to cancel the disturbance, and this is referred to as the internal model principle [7], [1]. This desired control input may be generated by an internal model or sometimes by disturbance observers, depending on the formulation of

Manuscript received September 29, 2014; revised December 15, 2014 and February 10, 2015; accepted May 7, 2015.

Copyright (C)2015 IEEE. Personal use of this material is permitted. However, permission to use this material for any other purposes must be obtained from the IEEE by sending a request to pubs-permissions@ieee.org.

Zhengtao Ding is with School of Electrical and Electronic Engineering, University of Manchester, Sackville Street Building, Manchester M13 9PL, United Kingdom. Email: zhengtao.ding@ manchester.ac.uk the disturbance rejection problem [8], [9]. It is well known that output regulation design also deals with the rejection of deterministic disturbances [10], [11]. In the formulation, output regulation usually combines the rejection and the output tracking problem, while the measurement used for disturbance rejection problem usually does not contain the deterministic disturbance directly [12]. Typical results for output regulation and disturbance rejection may be found in the references [1], [10], [11], [6]. One common design method for disturbance rejection is to estimate the disturbances from the state or output measurements, and then use the estimate disturbance to cancel the influence of the disturbance in the system. Many results have been published in this area for linear and nonlinear systems [13], [14], [15], [16], [4], [2], [17], [18], [19], [20], of which some are applied successfully to various situations [21], [2], [22], [23].

Recently, many control applications are related to dynamic systems that are connected by a communication network. A more specific topic in this area is to coordinate individual dynamic systems with the same or similar dynamics to perform a common control task, and this topic is commonly known as consensus control. In the formulation of consensus control problems, there are two types of problems, consensus without leader and leader-follower consensus [24], [25]. The important progress in consensus control was due to the application of graph theory, in particular, the Laplacian matrix of the network connection, to the control design of interconnected dynamic systems [24], [26], [27], [28], [29]. The early results concentrate on consensus issues of simple subsystem dynamics such as single integrators, or linear systems under directed connection graph [28] while the recent results have been obtained for general linear systems under undirected graph [27] and some for nonlinear systems [29]. Even for output regulation, there have seen some recent publications on consensus output regulation, often in the set-up with a pinning control or some information of the desired reference, possibly from an exosystem [30], [31], [32]. There is a lack of systematic study on disturbance rejection via disturbance observers for consensus control. The aim of this paper is to bridge this gap.

One difference of consensus control from the control design of individual systems is that consensus control design uses the relative state or output information. If the actual state or output of individual systems were available, the existing techniques could be applied to simplify the input-output dynamics to single integrators and then consensus control would be straightforward. The existing design techniques for disturbance observer design of individual systems cannot be 
directly applied, due to the restriction of the relative information. Certain features in the individual system dynamics are first explored, and a disturbance observer design is then proposed for a general network connection described by a directed graph. This proposed design uses the most common assumption in the literature about the network connection, that is, there exists a spanning tree in a directed connection graph, more challenging than control with an undirected connection graph. The proposed disturbance observer uses relative state information gathered from the subsystems in the neighbourhood, and the observer gains depend on the network connectivity, in the same way as most of consensus control design. A fully distributed scheme is then proposed to relax this requirement using adaptive control techniques to estimate the connectivity of the connection graph, inspired by the fully distributed consensus control design [33]. For the conceptual clarity, the proposed algorithm is presented for network connections with undirected connection graphs. Subsequently, a scheme for consensus disturbance rejection is also proposed for the leader-follower case. Because the proposed scheme is based on the relative state information, the disturbances that do not cause a difference in the common trajectory of the subsystems will not be rejected. An example of rejecting input disturbances in flight formation control is used to demonstrate the proposed design method. It can be seen from the simulation result that the flight formation is maintained under the disturbances, but the disturbances have an impact in the common trajectories.

\section{Problem Statement}

In this paper, we consider a set of $N$ linear subsystems, of which the subsystem dynamics are described by

$$
\dot{x}_{i}=A x_{i}+B u_{i}+D w_{i},
$$

for $i=1, \ldots N$, where $A \in \mathbb{R}^{n \times n}, B \in \mathbb{R}^{n \times m}, D \in \mathbb{R}^{n \times s}$ are constant matrices, $x_{i} \in \mathbb{R}^{n}$ is the state vector, with $n$ a known positive constant integer denoting the order of the subsystems, $u_{i} \in \mathbb{R}^{m}$ the input, and $w_{i} \in \mathbb{R}^{s}$ is a disturbance that is generated by an exosystem

$$
\dot{w}_{i}=S w_{i}
$$

with $S \in \mathbb{R}^{s \times s}$ being a known constant matrix.

The connections among the subsystems are specified by a graph $\mathcal{G}$ which consists of a set of vertices denoted by $\mathcal{V}$ and a set of edges denoted by $\mathcal{E}$. A vertex represents a subsystem, and each edge represents a connection. Associated with the graph, its adjacency matrix $\mathcal{A}$ with elements $a_{i j}$ denotes the connections such that $a_{i j}=1$ if there is a path from subsystem $j$ to subsystem $i$, and $a_{i j}=0$ otherwise. We define the Laplacian matrix $L$ in the normal way as $l_{i i}=\sum_{j=1}^{N} a_{i j}$ and $l_{i j}=-a_{i j}$ when $i \neq j$. The connection graph is undirected if $a_{i j}=a_{j i}$, i.e., the adjacency matrix is symmetric. Otherwise, the connection graph is directed. Clearly, consensus control with directed graph is more challenging than that with the undirected, as the directed graph includes the undirected graph as a special case.

The consensus disturbance rejection problem is to design control inputs using the relative state information collected from the network to ensure that all the subsystem states converge to a common trajectory when the subsystems are subject to different disturbances. The control inputs are based on the consensus disturbance observers that estimate the part of disturbances in the subsystems that cause differences from a common trajectory.

We make three assumptions about the dynamics of the subsystems and the connections between the subsystems.

Assumption 1. The disturbance is matched, i.e., there exist a matrix $F \in \mathbb{R}^{s \times s}$ such that $D=B F$.

Assumption 2. The eigenvalues of the exosystem matrix $S$ are distinct, and on the imaginary axis, and the pair $(S, D)$ is observable.

Assumption 3. The Laplacian matrix of the connection graph has a single eigenvalue at 0 .

From Assumption 3, we know that the Laplacian matrix $L$ only has one eigenvalue at 0 , and all other eigenvalues are with positive real part. In this case, the least positive real part is also known as the connectivity of the network, denoted by $\alpha$.

Remark 1: Assumption 1 on the matched disturbance could be relaxed in some circumstances, because unmatched disturbances under uncertain conditions may be converted to the matched ones based on output regulation theory [8]. The assumption on the eigenvalues of $S$ is commonly used for disturbance rejection and output regulation. It allows the disturbances be sinusoidal functions and constants. Other functions may be approximated by sinusoidal functions with a bias. It is also common in consensus and cooperative control to assume the common exosystem dynamics, to reflect common dynamics and environment of the subsystems [30]. From an engineering point of view, if the exosystem dynamics reflect certain unmodelled dynamics of the subsystems, it would be natural to assume that they are common in consensus control, as one would expect that the subsystem dynamics are common. The assumption on the observability of $(S, D)$ is reasonable, as any unobservable components would have no impact on system states.

Remark 2: The condition specified in Assumption 3 is satisfied if the connection graph contains a directed spanning tree.

\section{Preliminary Results}

When the disturbance $w$ is known, the disturbance rejection is fairly straightforward by adding the term $-F w_{i}$ in the control input $u_{i}$. The key issue is to estimate $w_{i}$ using the relative state information. At least we would expect that the disturbance state $w_{i}$ is observable from the system state measurement $x_{i}$. For this, we have the following lemma.

Lemma 1: If the pair $(S, D)$ is observable, the pair $\left(A_{H}, H\right)$ is observable, with $A_{H}=\left[\begin{array}{cc}A & D \\ 0 & S\end{array}\right]$ and $H=$ $\left[\begin{array}{ll}I & 0\end{array}\right]$.

Proof. Let us establish the result by seeking a contradiction. Suppose that $\left(A_{H}, H\right)$ is not observable, for any eigenvalue 
of $A_{H}, \lambda_{j}$, the matrix

$$
\left[\begin{array}{cc}
\lambda_{j} I-A & -D \\
0 & \lambda_{j} I-S \\
I & 0
\end{array}\right]
$$

is rank deficient, i.e., there exists a non-zero vector $v=$ $\left[v_{1}^{T}, v_{2}^{T}\right]^{T} \in \mathbb{R}^{(n+s)}$ such that

$$
\left[\begin{array}{cc}
\lambda_{j} I-A & -D \\
0 & \lambda_{j} I-S \\
I & 0
\end{array}\right]\left[\begin{array}{l}
v_{1} \\
v_{2}
\end{array}\right]=0 .
$$

This implies that

$$
\begin{aligned}
v_{1} & =0 \\
{\left[\begin{array}{c}
-D \\
\lambda_{j} I-S
\end{array}\right] v_{2} } & =0 .
\end{aligned}
$$

Since $v_{1}=0$, we have $v_{2} \neq 0$, which implies, together with (3), that $(S, D)$ is not observable. This is an contradiction, and therefore $\left(A_{H}, H\right)$ must be observable.

Since $\left(A_{H}, H\right)$ is observable, there exists a positive definite matrix $Q$ that satisfies

$$
Q A_{H}+A_{H}^{T} Q-2 H^{T} H<0 .
$$

We also have a result for the Laplacian matrices of undirected graphs.

Lemma 2: If the Laplacian matrix $L$ satisfies Assumption 3 , and is symmetric, then the following result holds:

$$
\sum_{i=1}^{N} \sum_{j=1}^{N} l_{i j} x_{i}^{T} x_{j} \geq \alpha \sum_{i=1}^{N} x_{i}^{T} x_{i}
$$

for any $x_{i} \in \mathbb{R}^{n}$, for $i=1, \ldots, N$, that satisfies $\sum_{i=1}^{N} x_{i}=0$. Proof. A similar version has been used in literature. As the proof is fairly short, we include it here. Let $x=$ $\left[x_{1}^{T}, \ldots, x_{N}^{T}\right]^{T}$. We can write

$$
\Delta:=\sum_{i=1}^{N} \sum_{j=1}^{N} l_{i j} x_{i}^{T} x_{j}=x^{T}\left(L \otimes I_{n}\right) x
$$

Since $L$ is symmetric, we have $L=U^{T} D U$ with $U$ being a unitary matrix and $D$ being a diagonal matrix. Since $L$ has only one eigenvalue at zero and the connectivity is $\alpha$, we can arrange $D=\operatorname{diag}\left(d_{1}, d_{2}, \ldots, d_{N}\right)$ with $d_{1}=0$ and $d_{i} \geq$ $\alpha$ for $i>1$. Hence we have the first row of $U$ as $U_{(1)}=$ $[1, \ldots, 1] / \sqrt{N}$. Clearly we have $U_{(1)} \otimes I_{n} x=0$ and we can partition $U \otimes I_{n} x=\left[\begin{array}{l}0 \\ y\end{array}\right]$ with $y \in \mathbb{R}^{n(N-1)}$. This leads to

$$
\begin{aligned}
\Delta & =x^{T}\left(U^{T} \otimes I_{n}\right)\left(D \otimes I_{n}\right)\left(U \otimes I_{n}\right) x \\
& =\left[0, y^{T}\right]\left(D \otimes I_{n}\right)\left[0, y^{T}\right]^{T} \\
& \geq \alpha\|y\|^{2}=\alpha\|x\|^{2} .
\end{aligned}
$$

The last equality in the above follows from the fact that $U$ is a unitary matrix. This completes the proof.

For the following lemma, note that an M-matrix is a square matrix whose off-diagonal elements are negative and all principal minors are positive [34].

Lemma 3: If the spanning tree implied by Assumption 3 is with the node 1 as the root, the Laplacian matrix can be partitioned as $L=\left[\begin{array}{cc}0 & 0_{1 \times(N-1)} \\ L_{0 c} & \bar{L}\end{array}\right]$, with the matrix $\bar{L} \in \mathbb{R}^{(N-1) \times(N-1)}$ being a non-singular M-matrix, and there exists a positive diagonal matrix $P=\operatorname{diag}\left\{p_{1}, \ldots, p_{N-1}\right\}$ such that

$$
P \bar{L}+\bar{L}^{T} P \geq r_{0} I
$$

for some positive constant $r_{0}$.

Proof. This result has been used in literature such as [35]. It is easy to see that $\bar{L}$ is a non-singular M-matrix from the structure of $L$ [34], and the existence of $\bar{L}$ for (8) follows from a theorem in [34]. It is also shown in [34] that $P$ can be generated by letting $P=(\operatorname{diag}(x))^{-1}$ with $x \in \mathbb{R}^{N-1}$ and $x=\bar{L}^{-1}[1, \ldots, 1]^{T}$.

Note that $\bar{L}$ is formed by elements $l_{i j}$ for $i, j=2, \ldots, N$. If we denote the elements of $\bar{L}$ by $\bar{l}_{i j}$ with $i, j=1, \ldots, N-1$, we have $\bar{l}_{i j}=l_{(i+1)(j+1)}$.

\section{Disturbance REJECTION WITH DIRECTED GRAPH}

In this section, we consider consensus disturbance rejection with directed graphs. Further to the condition specified in Assumption 3, we state the following condition.

Assumption 4. The minimum value of the network connectivity $\alpha$ is known.

Note that the connectivity can be easily calculated from the Laplacian matrix of a network connection. By doing so, it does require all the elements of $a_{i j}$ which are not restricted to the neighbourhood of a subsystem. In the next section, the assumption on the network connectivity is relaxed by a fully distributed consensus disturbance rejection scheme.

The disturbance rejection design is based on disturbance estimation and rejection. The estimation is based on the relative state information. Define, for $i=1, \ldots, N$,

$$
\zeta_{i}=\sum_{j=1}^{N} a_{i j}\left(x_{i}-x_{j}\right)
$$

From the definition of $l_{i j}$, it is easy to see that $\zeta_{i}=$ $\sum_{j=1}^{N} l_{i j} x_{j}$. The disturbance estimation and rejection method proposed in this paper will be based on the signals $\zeta_{i}$.

The control input for disturbance rejection is designed as follows

$$
u_{i}=-K \chi_{i}-F z_{i},
$$

where $\chi_{i} \in \mathbb{R}^{n}$ and $z_{i} \in \mathbb{R}^{s}$ are generated by

$$
\begin{aligned}
& \dot{\chi}_{i}=(A-B K) \chi_{i}+c G_{x}\left(\zeta_{i}-\sum_{j=1}^{N} l_{i j} \chi_{j}\right), \\
& \dot{z}_{i}=S z_{i}+c G_{w}\left(\zeta_{i}-\sum_{j=1}^{N} l_{i j} \chi_{j}\right),
\end{aligned}
$$

where $c \geq 1 / \alpha$ is a positive real constant, $K$ is a constant gain matrix with a proper dimension such that $(A-B K)$ is Hurwitz, and $\left[G_{x}^{T}, G_{w}^{T}\right]^{T}:=G=Q^{-1} H^{T}$. Note that (11) is designed as a state observer as only the relative state information is available, and (12) is designed as a consensus disturbance observer. 
Let

$$
e_{i}=\left[\begin{array}{c}
x_{i}-\chi_{i} \\
w_{i}-z_{i}
\end{array}\right]
$$

From (1), (2), (11) and (12), we can establish that

$$
\dot{e}_{i}=A_{h} e_{i}+c G \sum_{j=1}^{N} l_{i j} H e_{j} .
$$

Let $e=\left[e_{i}^{T}, \ldots, e_{N}^{T}\right]^{T}$. The dynamics of $e$ can be obtained as

$$
\dot{e}=\left(I_{N} \otimes A_{H}-c L \otimes G H\right) e .
$$

Let us define $r \in \mathbb{R}^{N}$ as the left eigenvector of $L$ corresponding to the eigenvalue at 0 , that is, $r^{T} L=0$, and furthermore, we set $r^{T} \mathbf{1}=1$ with $\mathbf{1}=[1, \ldots, 1]^{T} \in \mathbb{R}^{N}$.

Based on the vector $r$, we can introduce a state transformation $\xi_{i}=e_{i}-\sum_{j=1}^{N} r_{j} e_{j}$ for $i=1, \ldots, N$. With $\xi=\left[\xi_{1}^{T}, \ldots \xi_{N}^{T}\right]^{T}$, the state transformation is given by

$$
\begin{aligned}
\xi & =e-\left(\left(\mathbf{1} r^{T}\right) \otimes I_{n+s}\right) e \\
& =M \otimes I_{n+s} e,
\end{aligned}
$$

where $M=I_{N}-\mathbf{1} r^{T}$.

It can be shown that the rank of $M$ is $N-1$ with one eigenvalue at 0 and others at 1 . The eigenvector corresponding to the eigenvalue at 0 is $\mathbf{1}$, and then, $\xi=0$ implies that $e_{1}=e_{2}=\ldots=e_{N}$.

For each subsystem, we have

$$
\begin{aligned}
\dot{x}_{i} & =A x_{i}-B K \chi_{i}+B F\left(w_{i}-z_{i}\right) \\
& =(A-B K) x_{i}+B[K, F] e_{i} .
\end{aligned}
$$

Hence $\lim _{t \rightarrow \infty} \xi(t)=0$ ensures $\lim _{t \rightarrow \infty} x_{1}(t)=$ $\lim _{t \rightarrow \infty} x_{2}(t)=\ldots=\lim _{t \rightarrow \infty} x_{N}(t)$, the solution of the consensus disturbance rejection problem.

Theorem 4: For the network-connected systems with the subsystem dynamics (1), the control inputs (10) with the state and disturbance observers (11) and (12) solve the consensus disturbance rejection problem under Assumptions 1 to 4 .

Proof. The dynamics of $\xi$ can then be obtained as

$$
\dot{\xi}=\left(I_{N} \otimes A_{H}-c L \otimes G H\right) \xi .
$$

To exploit the structure of $L$, let us introduce another state transformation

$$
\eta=T^{-1} \otimes I_{n+s} \xi,
$$

where $T$ is the transformation such that $T^{-1} L T=J$ with $J$ being in the real Jordan canonical form, as shown in Lemma 3 of [29].

Its dynamics are obtained as

$$
\dot{\eta}=\left(I_{N} \otimes A_{H}-c J \otimes G H\right) \eta .
$$

Hence the consensus disturbance rejection problem is solved by showing that $\eta$ converges to zero. Also note that the transformations shown in (19) and (16) also imply that

$$
\eta_{1}=r^{T} \otimes I_{n+s} \xi=\left(r^{T} M\right) \otimes I_{n+s} e \equiv 0 .
$$

The matrix $J$ is in the real Jordan canonical form with the first diagonal element equalling 0 . Assume that $\lambda_{i}$ is a single real eigenvalue of $L$. From (20), the dynamics of $\eta_{i}$ is obtained as

$$
\begin{aligned}
\dot{\eta}_{i} & =\left(A_{H}-c \lambda_{i} G H\right) \eta_{i} \\
& =\left(A_{H}-c \lambda_{i} Q^{-1} H^{T} H\right) \eta_{i} .
\end{aligned}
$$

Let

$$
V_{i}=\eta_{i}^{T} Q \eta_{i}
$$

From (20), we have

$$
\begin{aligned}
\dot{V}_{i} & =\eta_{i}^{T}\left(Q A_{H}+A_{H}^{T} Q-2 c \lambda_{i} H^{T} H\right) \eta_{i} \\
& \leq \eta_{i}^{T}\left(Q A_{H}+A_{H}^{T} Q-2 H^{T} H\right) \eta_{i} \\
& \leq-\epsilon\left\|\eta_{i}\right\|^{2}
\end{aligned}
$$

for some positive real constant $\epsilon$, because of $c \lambda_{i} \geq c \alpha \geq 1$.

Following the procedures in the proof of Corollary 3.4 in [29], we can establish results similar to (23) for complex eigenvalues, multiple eigenvalues and multiple complex eigenvalues. Let

$$
V_{\eta}=\sum_{i=2}^{N} V_{i}
$$

and it can then be obtained that

$$
\dot{V} \leq-\epsilon\|\eta\|^{2} .
$$

Therefore $\eta$ converges to 0 exponentially.

\section{Distributed CONSENSUS DisturbanCE REJECTION}

The control in the previous section takes the relative state information from the subsystems in the neighbourhood, and needs the knowledge of the connectivity. This is a common assumption in most of the consensus control design. However, the connectivity is defined as the minimum positive real part of the eigenvalues of the Laplacian matrices. The evaluation of this value does need the Laplacian matrix or the adjacency matrix, i.e, the entire $a_{i j}$ for $i, j=1, \ldots, N$. Hence the connectivity is a global information of the network connection. The proposed disturbance rejection algorithm in the previous section is not fully distributed in this sense.

In this section, we shall present a design that only uses the local connection information, $a_{i j}$ values for the $i$ th subsystem, and the relative state information within the neighbourhood. This fully distributed consensus disturbance rejection is for connections with undirected graph. Thus we have the following assumption.

Assumption 5. The network connection graph is undirected.

Adaptive control technique is used to deal with unknown connectivity. The control input is designed in the same way as shown in (10)

$$
u_{i}=-K \chi_{i}-F z_{i} .
$$

But the estimated state and disturbance $\chi_{i}$ and $z_{j}$ are now generated by

$$
\begin{aligned}
\dot{\chi}_{i} & =(A-B K) \chi_{i}+G_{x} \sum_{j=1}^{N} c_{i j} a_{i j}\left(\rho_{i}-\rho_{j}\right), \\
\dot{z}_{i} & =S z_{i}+G_{w} \sum_{j=1}^{N} c_{i j} a_{i j}\left(\rho_{i}-\rho_{j}\right),
\end{aligned}
$$


where $\rho_{i}:=x_{i}-\chi_{i}$, and $c_{i j}$ are adaptive parameters generated by

$$
\dot{c}_{i j}=\left(\rho_{j}-\rho_{i}\right)^{T}\left(\rho_{j}-\rho_{i}\right)
$$

with $c_{i j}(0)=c_{j i}(0)$.

Note that the only difference in the control design, compared with the one shown in the previous section, is that the observer gain parameter $c$ in the previous design is replaced by adaptive parameters $c_{i j}$. The setting $c_{i j}(0)=c_{j i}(0)$ for the initial values is to keep the symmetry of the adaptive parameters.

The adaptive parameters also make a difference in stability analysis. The dynamics of $e_{i}$ are now obtained as

$$
\dot{e}_{i}=A_{H} e_{i}+G \sum_{j=1}^{N} c_{i j} a_{i j} H\left(e_{j}-e_{i}\right)
$$

where $e_{i}$ follows the same definition as in the previous section. Let us re-define $\xi_{i}$ as $\xi_{i}=e_{i}-\frac{1}{N} \sum_{j=1}^{N} e_{j}$. It can be obtained that

$$
\dot{\xi}_{i}=A_{H} \xi_{i}+G \sum_{j=1}^{N} c_{i j} a_{i j} H\left(\xi_{j}-\xi_{i}\right) .
$$

To obtain the above expression, we have observed that $\left(e_{j}-\right.$ $\left.e_{i}\right)=\left(\xi_{j}-\xi_{j}\right)$ and $\frac{1}{N} G \sum_{j=1}^{N} \sum_{k=1}^{N} c_{j k} a_{j k} H\left(e_{j}-e_{k}\right)=0$. The consensus disturbance rejection problem is solved if we can show that $\xi_{i}$ converges to 0 , for $i=1, \ldots, N$, in the same way as discussed in the previous section.

Theorem 5: For the network-connected system with the subsystem dynamics (1), the control inputs (26) with the state and disturbance observers (27) and (28) solve the distributed consensus disturbance rejection problem under Assumptions 1 to 3 and 5 .

Proof. Using the notation $\xi_{i}$, the adaptive laws can be written as

$$
\dot{c}_{i j}=\left(\xi_{j}-\xi_{i}\right)^{T} H^{T} H\left(\xi_{j}-\xi_{i}\right) .
$$

Let

$$
V_{i}=\xi_{i}^{T} Q \xi_{i} .
$$

From the dynamics of $\xi_{i}$ and $c_{i j}$ shown earlier, we have

$$
\begin{aligned}
\dot{V}_{i}= & \xi_{i}^{T}\left(Q A_{H}+A_{H}^{T} Q\right) \xi_{i} \\
& +2 \xi_{i}^{T} Q G \sum_{j=1}^{N} c_{i j} a_{i j} H\left(\xi_{j}-\xi_{i}\right) \\
= & \xi_{i}^{T}\left(Q A_{H}+A_{H}^{T} Q-2 H^{T} H\right) \xi_{i}+2 \xi_{i}^{T} H^{T} H \xi_{i} \\
& +2 \xi_{i}^{T} H^{T} H \sum_{j=1}^{N} c_{i j} a_{i j}\left(\xi_{j}-\xi_{i}\right) \\
\leq & -\epsilon\left\|\xi_{i}\right\|^{2}+2 \xi_{i}^{T} H^{T} H \xi_{i} \\
& +2 \xi_{i}^{T} H^{T} H \sum_{j=1}^{N} c_{i j} a_{i j}\left(\xi_{j}-\xi_{i}\right),
\end{aligned}
$$

where $\epsilon$ is a positive real constant.

Let

$$
V_{c}=\frac{1}{2} \sum_{i=1}^{N} \sum_{j=1}^{N} a_{i j}\left(c_{i j}-\frac{1}{\alpha}\right)^{2}
$$

Its derivative is obtained as

$$
\begin{aligned}
\dot{V}_{c} & =\sum_{i=1}^{N} \sum_{j=1}^{N} a_{i j}\left(c_{i j}-\frac{1}{\alpha}\right)\left(\xi_{j}-\xi_{i}\right)^{T} H^{T} H\left(\xi_{j}-\xi_{i}\right) \\
& =-2 \sum_{i=1}^{N} \sum_{j=1}^{N} a_{i j}\left(c_{i j}-\frac{1}{\alpha}\right) \xi_{i}^{T} H^{T} H\left(\xi_{j}-\xi_{i}\right),
\end{aligned}
$$

where the last equation is obtained by using $c_{i j}=c_{j i}$. We can further expand the terms in (36) to obtain that

$$
\begin{aligned}
\dot{V}_{c}= & -2 \sum_{i=1}^{N} \sum_{j=1}^{N} a_{i j} c_{i j} \xi_{i}^{T} H^{T} H\left(\xi_{j}-\xi_{i}\right) \\
& -2 \frac{1}{\alpha} \sum_{i=1}^{N} \sum_{j=1}^{N} l_{i j} \xi_{i}^{T} H^{T} H \xi_{j} \\
\leq & -2 \sum_{i=1}^{N} \sum_{j=1}^{N} a_{i j} c_{i j} \xi_{i}^{T} H^{T} H\left(\xi_{j}-\xi_{i}\right) \\
& -2 \sum_{i=1}^{N} \xi_{i}^{T} H^{T} H \xi_{i},
\end{aligned}
$$

where the inequality is obtained by viewing $H \xi_{i}$ as $x_{i}$ in Lemma 2.

Let

$$
V=\sum_{i=1}^{N} V_{i}+V_{c} .
$$

From the results shown in (34) and (37), we have

$$
\dot{V} \leq \sum_{i=1}^{N}-\epsilon\left\|\xi_{i}\right\|^{2} .
$$

Hence we can conclude that all the variables in the entire system are bounded, and $\xi_{i} \in L_{2} \cap L_{\infty}$ for $i=1, \ldots, N$. Since the derivatives of $\xi_{i}$ are bounded, we conclude that $\xi_{i}$ for $i=1, \ldots, N$ converge to zero, by Babalat's lemma [36]. This completes the proof.

\section{DisturbanCE REJECTION IN LEADER FOLLOWER FORMAT}

In the previous sections, control schemes are proposed for disturbance rejection in consensus control without a leader, i.e., the final common value depends on all the subsystems involved in the network. Another form of consensus control is that one subsystem is identified as the leader, and other subsystems are controlled to follow this leader. In this section, we propose a control design for consensus disturbance rejection with a leader. Without loss of generality, we assume the subsystem at node 1 in the connection graph is the leader. Based on this, we have an assumption about the network connection.

Assumption 6. There exists a spanning tree in the connection graph with the subsystem at node 1 as the root.

When a network contains a spanning tree, the corresponding Laplacian matrix will only have one eigenvalue at 0 , and therefore Assumption 6 supersedes Assumption 3. 
With the subsystem 1 as the leader, the control objective is to ensure the state variables of other subsystems to follow the state of the leader, $x_{1}$, under the disturbances.

Let

$$
\bar{x}_{i}=x_{i+1}-x_{1}
$$

for $i=1, \ldots, N-1$. As a common practice in leader-follower consensus control, the leader does not take any control from consensus control design, i.e., we have $u_{1}=0$. The dynamics of $\bar{x}_{i}$, for $i=1, \ldots, N-1$, are described by

$$
\begin{aligned}
\dot{\bar{x}}_{i} & =A \bar{x}_{i}+B \bar{u}_{i}+D \bar{w}_{i}, \\
\dot{\bar{w}}_{i} & =S \bar{w}_{i},
\end{aligned}
$$

where $\bar{w}_{i}=w_{i+1}-w_{1}$ and $\bar{u}_{i}=u_{i+1}$.

We still use the signal $\zeta_{i}$ defined in (9) of Section 3 for the disturbance observer and control design. To express $\zeta_{i}$ in $\bar{x}_{i}$, we have, for $i=2, \ldots, N$,

$$
\begin{aligned}
\zeta_{i} & =\sum_{j=2}^{N} l_{i j} x_{j}+l_{i 1} x_{1}=\sum_{j=2}^{N} l_{i j} x_{j}-\sum_{j=2}^{N} l_{i j} x_{1} \\
& =\sum_{j=2}^{N} l_{i j} \bar{x}_{j-1}=\sum_{j=1}^{N-1} \bar{l}_{(i-1) j} \bar{x}_{j} .
\end{aligned}
$$

For the notational convenience, we let $\bar{\zeta}_{i}=\zeta_{i+1}$. Hence we have

$$
\bar{\zeta}_{i}=\sum_{j=1}^{N-1} \bar{l}_{i j} \bar{x}_{j} .
$$

The control input for disturbance rejection in the leaderfollower set-up is designed as follows, for $i=1, \ldots, N-1$,

$$
\bar{u}_{i}=-K \chi_{i}-F z_{i}
$$

where $\chi_{i}$ and $z_{i}$ are generated by

$$
\begin{aligned}
& \dot{\chi}_{i}=(A-B K) \chi_{i}+c G_{x}\left(\bar{\zeta}_{i}-\sum_{j=1}^{N-1} \bar{l}_{i j} \chi_{j}\right), \\
& \dot{z}_{i}=S z_{i}+c G_{w}\left(\bar{\zeta}_{i}-\sum_{j=1}^{N-1} \bar{l}_{i j} \chi_{j}\right),
\end{aligned}
$$

where $c \geq 2 p_{\max } / r_{0}$ is a positive real constant with $p_{\max }=$ $\max \left\{p_{1}, \ldots, p_{N-1}\right\}$, and we still use $\left[G_{x}^{T}, G_{w}^{T}\right]^{T}=Q^{-1} H^{T}$.

Let

$$
\bar{e}_{i}=\left[\begin{array}{c}
\bar{x}_{i}-\chi_{i} \\
\bar{w}_{i}-z_{i}
\end{array}\right]
$$

for $i=1, \ldots, N-1$. The dynamics of $\bar{e}_{i}$ can be obtained as

$$
\dot{\bar{e}}_{i}=A_{h} \bar{e}_{i}+c G \sum_{j=1}^{N-1} \bar{l}_{i j} H \bar{e}_{j} .
$$

Let $\bar{e}=\left[\bar{e}_{1}^{T}, \ldots, \bar{e}_{N-1}^{T}\right]^{T}$. The dynamics of $\bar{e}$ can be obtained as

$$
\dot{\bar{e}}=\left(I_{N-1} \otimes A_{H}-c \bar{L} \otimes G H\right) \bar{e} .
$$

It can be seen that the dynamics of $\bar{e}$ in (48) are in the same format as the dynamics shown in (15). But in this case, $\bar{L}$ is a non-singular matrix, and the stability analysis will be different. In this leader-follower case, we will establish that all the $\bar{e}_{i}$, for $i=1, \ldots, N-1$, converge to 0 , instead of showing they converge to the same values as in the leaderless consensus control case.

Theorem 6: For the network-connected systems with the subsystem dynamics (1), the control inputs (43) with the disturbance observers (44) and (45) solve the consensus disturbance rejection problem in leader-follower case under Assumptions 1, 2 and 6.

Proof. Let

$$
V_{e}=\bar{e}^{T}(P \otimes Q) \bar{e} .
$$

By the result in Lemma 3, its derivative can be obtained as

$$
\begin{aligned}
\dot{V}_{e}= & \bar{e}^{T}\left[P \otimes\left(Q A_{H}+A_{H}^{T} Q\right)\right. \\
& \left.-c\left(P \bar{L}+\bar{L}^{T} P\right) \otimes H^{T} H\right] \bar{e} \\
\leq & \bar{e}^{T}\left[P \otimes\left(Q A_{H}+A_{H}^{T} Q\right)-c r_{0} I_{N-1} \otimes H^{T} H\right] \bar{e} \\
\leq & \bar{e}^{T}\left[P \otimes\left(Q A_{H}+A_{H}^{T} Q-2 H^{T} H\right)\right] \bar{e} \\
\leq & -\epsilon \bar{e}^{T} \bar{e},
\end{aligned}
$$

where $\epsilon$ is a positive real number. Therefore, we can conclude that $\bar{e}$ converges to zero exponentially. As the closed-loop dynamics of $\bar{x}_{i}$ can be written as

$$
\dot{\bar{x}}_{i}=(A-B K) \bar{x}_{i}+[B K, D] \bar{e}_{i},
$$

we further conclude that $\bar{x}_{i}$ converges to zero exponentially for $i=1, \ldots, N-1$.

\section{EXAMPLE}

In this section, an example is used to demonstrate the potential applications of the proposed consensus disturbance rejection methods. We consider an example of consensus disturbance rejection of a possible vibration in control surface for formation control of UAVs. The formation control of YF22 research UAVs was presented in [37] where the longitudinal dynamics are described by

$$
\begin{aligned}
{\left[\begin{array}{c}
\dot{V} \\
\dot{\alpha} \\
\dot{q} \\
\dot{\theta}
\end{array}\right]=} & {\left[\begin{array}{rrrr}
-0.284 & -23.096 & 0 & -0.171 \\
0 & -4.117 & 0.778 & 0 \\
0 & -33.884 & -3.573 & 0 \\
0 & 0 & 1 & 0
\end{array}\right] } \\
& \times\left[\begin{array}{c}
V \\
\alpha \\
q \\
\theta
\end{array}\right]+\left[\begin{array}{r}
20.168 \\
0.544 \\
-39.085 \\
0
\end{array}\right] i_{H},
\end{aligned}
$$

where $V, \alpha, q$ and $\theta$ are the speed in $\mathrm{m} / \mathrm{s}$, angle of attack in degree, pitch rate in degree/s and pitch in degree, respectively, and $i_{H}$ is the stabilator incidence angle in degree. The existing control law [37] is set as $i_{H}=0.12 q+0.50 \theta$.

As shown in [37], the formation control has been introduced, and our task in this example is to reject harmonic disturbances in the input channel, which can represent an undesired vibration. Since the system model is linear in this operating point, we can add the control input designed by the proposed consensus disturbance rejection method to the existing control. For $i$ th system, we use $x_{i}$ to denote the state 
( $V, \quad \alpha, q, \quad \theta$ ), and $u_{i}$ for the additional control input generated for consensus disturbance rejection, and $[1,0] w_{i}$ as the input disturbance, of which $w_{i}$ is generated by $\dot{w}_{i}=$ $\left[\begin{array}{cc}0 & \omega \\ -\omega & 0\end{array}\right] w_{i}$, with $\omega$ as the vibration frequency in radian/s. Hence the input disturbance problem is formulated as the consensus disturbance rejection problem with equations (1) and (2) presented in Section II, and we can identify the system matrices as

$$
\begin{gathered}
A=\left[\begin{array}{rrrr}
-0.284 & -23.096 & 2.420 & 9.913 \\
0 & -4.117 & 0.843 & 0.272 \\
0 & -33.884 & -8.263 & -19.543 \\
0 & 1 & 0
\end{array}\right] \\
B=\left[\begin{array}{r}
20.168 \\
0.544 \\
-39.085 \\
0
\end{array}\right], \quad D=B\left[\begin{array}{ll}
1 & 0
\end{array}\right] .
\end{gathered}
$$

Note that the system matrix $A$ has considered the exiting feedback control. It can be checked that Assumptions 1 and 2 are satisfied, and the $F$ matrix is given by $F=\left[\begin{array}{ll}1 & 0\end{array}\right]$.

In this example, we demonstrate the consensus disturbance rejection method under the leader-follower set-up of $5 \mathrm{UAVs}$, whose connection graph is specified by the adjacency matrix

$$
\mathcal{A}=\left[\begin{array}{lllll}
0 & 0 & 0 & 0 & 0 \\
1 & 0 & 1 & 0 & 0 \\
0 & 0 & 0 & 0 & 1 \\
0 & 1 & 0 & 0 & 0 \\
0 & 0 & 0 & 1 & 0
\end{array}\right] .
$$

Note that the first row are all zeros, as the subsystem at node 1 is taken as the leader. Following the result shown in Lemma 3 , we obtain that $P=\operatorname{diag}\left\{\begin{array}{llll}1 / 4 & 1 / 7 & 1 / 5 & 1 / 6\end{array}\right\}$ and $r_{0}=0.0537$. With $p_{\max }=1 / 4$ and $2 p_{\max } / r_{0}=9.3110$, we set $c=10$ in the disturbance observer (45) and the relative state observer (44). The observer gains $G_{w}$ and $G_{x}$ follow the standard design procedures shown in section VI. Since $A$ is stable in this example, we set $K=0$ for the control input. Simulation study has been carried out with different disturbances for subsystems. The speeds of all the subsystems are shown in Figure 1, the all four states in Figure 2, and the control inputs in Figure 3.

From the results shown in the figures, it can be seen that all the five UAVs converge to the same set of states, even though they are under different disturbances, and therefore the formation is preserved. Also note that the consensus disturbance rejection only ensures the relative positions for the formation, not the complete rejection of the input disturbance in general, because the relative information is used. In this example, the disturbance of the leading UAV is not rejected at all. Such a complete rejection can be introduced when the information of the actual state is available, instead of the relative ones. In the case that there is no disturbance in the leading UAV, all the disturbances in the followers will be completed rejected by the proposed scheme.

\section{CONCLUSION}

In this paper, a number of fundamental design issues for consensus disturbance rejection have been addressed. System-

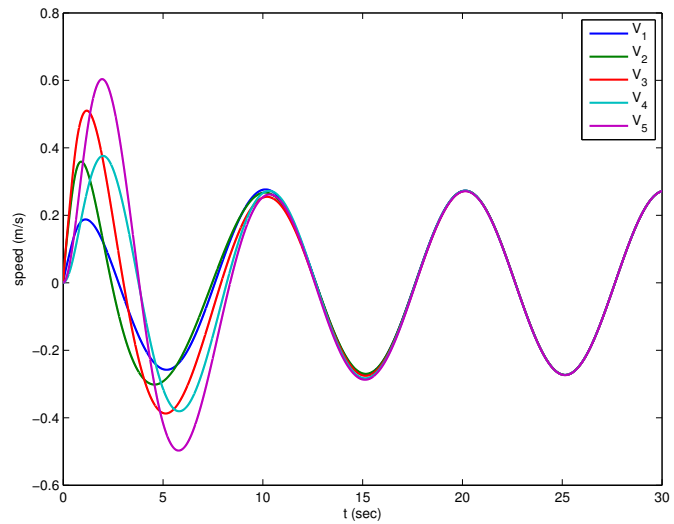

Fig. 1. The speed variations of UAVs.
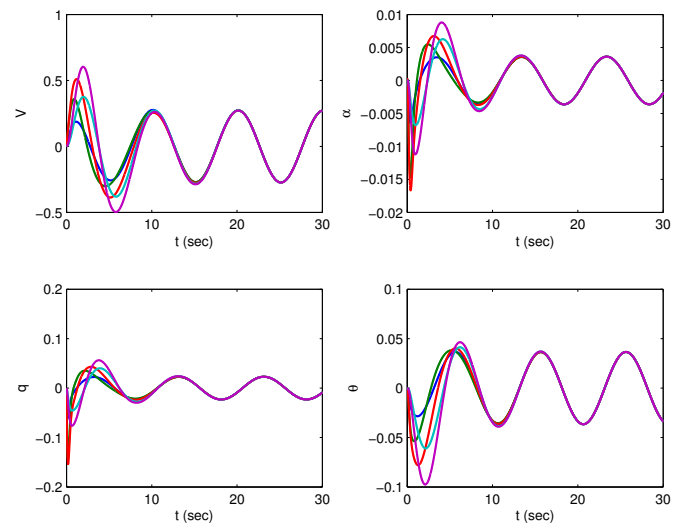

Fig. 2. Four states of UAV longitudinal control.

atic designs for consensus disturbance rejection have been proposed and analysed for network-connected multi-input linear systems using relative state information of the subsystems in the neighbourhood in leaderless and leader-follower consensus set-ups under some common assumptions of the network connections. Furthermore, in the leaderless consensus case, an adaptive scheme has been proposed to remove the requirement of the network connectivity in the disturbance observer design so that the proposed scheme is truly distributed in the sense that only the local information in the neighbourhood is used for the control design. Consensus disturbance rejection ensures the states of the subsystems converge to a consensus trajectory among all the subsystems, while this common trajectory may be influenced by the disturbances, as the use of relative state information restricts the rejection only to the part of the disturbances that causes differences among the subsystem trajectories. This is a difference from the disturbance rejection of individual systems due to the restriction of using relative state information. The partial rejection of disturbances for consensus control would consume less energy compared with the complete rejection of the disturbances for certain applications such as formation control. The design and analysis methods for consensus disturbance rejection are also different from the corresponding methods for standard consensus state feedback 


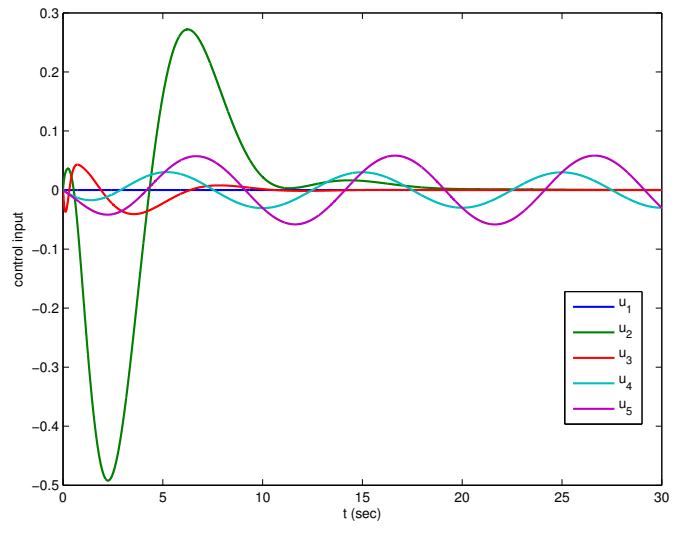

Fig. 3. The control inputs for consensus disturbance rejection.

control, due to the fact that the states for the disturbances are not controllable. The good simulation results suggest that the proposed methods have significant potentials in various engineering applications.

\section{REFERENCES}

[1] B. A. Francis, "The linear multivariable regulator problem," SIAM J. Contr. Optimiz., vol. 15, pp. 486-505, 1977.

[2] L. Guo and X.-Y. Wen, "Hierarchical anti-distance adaptive control for non-linear systems with composite disturbances and applications to missile systems," Transactions of the Institute of Measurement and Control, 2010.

[3] Z. Ding, "Global stabilization and disturbance suppression of a class of nonlinear systems with uncertain internal model," Automatica, vol. 39 , no. 3, pp. 471-479, 2003.

[4] L. Guo and W.-H. Chen, "Disturbance attenuation and rejection for systems with nonlinearity via dobc approach," Int. J. Robust and Nonlinear Control, vol. 15, no. 3, pp. 109-125, 2005.

[5] Z. Ding, "Asymptotic rejection of general periodic disturbances in output-feedback nonlinear systems," IEEE Trans. Autom. Control, vol. 51, no. 2, pp. 303-308, 2006.

[6] _ - "Output regulation of uncertain nonlinear systems with nonlinear exosystems," IEEE Trans. Autom. Control, vol. 51, no. 3, pp. 498-503, 2006.

[7] B. A. Francis and W. M. Wonham, "The internal model principle of control theory," Automatica, vol. 12, no. 5, pp. 457-465, 1976.

[8] A. Isidori, Nonlinear Control Systems, 3rd ed. Berlin: Springer-Verlag, 1995.

[9] S. Li, J. Yang, W.-H. Chen, and X. Chen, Disturbance Observer-based Control: Methods and Applications. CRC Press, 2014.

[10] A. Isidori and C. I. Byrnes, "Output regulation of nonlinear systems," IEEE Trans. Autom. Control, vol. 35, no. 2, pp. 131-140, 1990.

[11] J. Huang and W. J. Rugh, "On a nonlinear multivariable servomechanism problem," Automatica, vol. 26, no. 6, pp. 963-972, 1990.

[12] Z. Ding, "Universal disturbance rejection for nonlinear systems in output feedback form," IEEE Trans. Autom. Control, vol. 48, no. 7, pp. 1222 $1226,2003$.

[13] C. D. Johnson, "Accommodation of external disturbances in linear regulator and servomechanism problems." IEEE Trans. Autom. Control, vol. 16 , no. 6, pp. 635-644, 1971.

[14] W.-H. Chen, D. J. Ballance, P. J. Gawthrop, and J. O'Reilly, "A nonlinear disturbance observer for robotic manipulators," IEEE Trans. Ind. Electron., vol. 47, no. 4, pp. 932-938, 2000.

[15] W.-H. Chen, "Disturbance observer based control for nonlinear systems," IEEE/ASME Trans. Mechatronics, vol. 9, no. 4, pp. 706-710, 2004.

[16] M. Chen and W.-H. Chen, "Sliding mode control for a class of uncertain nonlinear system based on disturbance observer," Int. J. Adap. Control and Signal Proc., vol. 24, no. 1, pp. 51-64, 2010.

[17] Z. Ding, "Asymptotic rejection of unknown sinusoidal disturbances in nonlinear systems," Automatica, vol. 43, no. 1, pp. 174-177, 2007.
[18] - "Asymptotic rejection of unmatched general periodic disturbances with nonlinear lipschitz internal models," Int. J. Control, vol. 86, no. 2, pp. 210-221, 2013.

[19] J. Yang, S. Li, and X. Yu, "Sliding-mode control for systems with mismatched uncertainties via a disturbance observer," IEEE Trans. Ind. Electron., vol. 60, no. 1, pp. 160-169, 2013.

[20] E. Sariyildiz and K. Ohnishi, "On the explicit robust force control via disturbance observer," IEEE Trans. Ind. Electron., vol. 62, no. 3, pp. 1581-1589, 2015.

[21] X. Chen, J. Yang, S. Li, and Q. Li, "Disturbance observer based multi-variable control of ball mill grinding circuits," Journal of Process Control, vol. 19, no. 7, pp. 1205-1213, 2009.

[22] S. Li and Z. Liu, "Adaptive speed control for permanent-magnet synchronous motor system with variations of load inertia," IEEE Trans. Ind. Electron., vol. 56, no. 8, pp. 3050-3059, 2009.

[23] K. K. Tan, T. H. Lee, H. F. Dou, S. J. Chin, and S. Zhao, "Precision motion control with disturbance observer for pulsewidth-modulateddriven permanent-magnet linear motors," IEEE Trans. Magn., vol. 39, no. 3, pp. 1813-1818, 2003.

[24] R. Olfati-Saber and R. M. Murray, "Consensus problems in networks of agents with switching topology and time-delay," IEEE Trans. Autom. Control, vol. 49, no. 9, pp. 1520-1533, 2004.

[25] Y. Hong, J. Hu, and L. Gao, "Tracking control for multi-agent consensus with an active leader and variable topology," Automatica, vol. 42, no. 7 , pp. 1177-1182, 2006.

[26] R. Olfati-Saber, J. A. Fax, and R. M. Murray, "Consensus and cooperation in networked multi-agent systems," Proceedings of the IEEE, vol. 95, no. 1, pp. 215-233, 2007

[27] Z. Li, Z. Duan, G. Chen, and L. Huang, "Consensus of multiagent systems and synchronization of complex networks, a unified viewpoint," IEEE Trans. Circuits Syst. I, vol. 57, no. 1, pp. 213-224, 2010.

[28] J. Xi, Z. Shi, and Y. Zhong, "Output consensus analysis and design for high-order linear swarm systems: partial stability method," Automatica, vol. 48, no. 9, pp. 2335-2343, 2012.

[29] Z. Ding, "Consensus control of a class of lipschitz nonlinear systems," Int. J. Control, vol. 87, no. 11, pp. 2372-2382, 2014.

[30] Y. Su and J. Huang, "Cooperative adaptive output regulation for a class of nonlinear uncertain multi-agent systems with unknown leader," Systems \& Control Letters, vol. 62, no. 6, pp. 461-467, 2013.

[31] Z. Ding, "Consensus output regulation of a class of heterogeneous nonlinear systems," IEEE Trans. Autom. Control, vol. 58, no. 10, pp. 2648-2653, 2013.

[32] - "Adaptive consensus output regulation of a class of nonlinear systems with unknown high-frequency gain," Automatica, vol. 51, no. 1, pp. 348-355, 2015.

[33] Z. Li, W. Ren, X. Liu, and L. Xie, "Distributed consensus of linear multiagent systems with adaptive dynamic protocols," Automatica, vol. 49, no. 7, pp. 1986-1995, 2013.

[34] Z. Qu, Cooperative Control of Dynamical Systems. London: SpringerVerlag, 2009.

[35] Z. Li, G. Wen, Z. Duan, and W. Ren, "Designing fully distributed consensus protocols for linear multi-agent systems with directed graphs," IEEE Trans. Autom. Control, accepted for publication.

[36] Z. Ding, Nonlinear and Adaptive Control Systems. London: IET, 2013.

[37] J. Gu, B. Seanor, G. Campa, M. Napolitano, L. Rowe, S. Gururajan, and S. Wan, "Design and flight testing evaluation of formation control laws," IEEE Trans. Control Syst. Technol., vol. 14, no. 6, pp. 1105-1112, 2006.

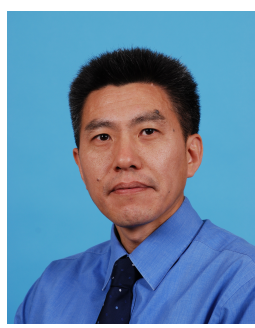

Zhengtao Ding (M'94-SM'03) graduated with BEng from Tsinghua University, Beijing, China. He then studied control engineering in the Control Systems Centre, UMIST, with MSc in systems and control and $\mathrm{PhD}$ in control systems. He joined University of Manchester in 2003 after having been a lecturer in Ngee Ann Polytechnic, Singapore for ten years, and he is now a senior lecturer in control engineering in School of Electrical and Electronic Engineering. His research interests are mainly focused on nonlinear and adaptive control theory and their applications. He has published a book entitled Nonlinear and Adaptive Control Systems, and a number of journal papers. He serves as an associate editor for IEEE Transactions on Automatic Control, Transactions of Institute of Measurement and Control, Control Theory and Technology, Mathematical Problems in Engineering, Unmanned Systems, and International Journal of Automation and Computing. 